Musées, Patrimoine et Culture scientifiques et techniques

$136 \mid 2011$

juillet - aôut 2011

\title{
Un exemple de conservation-restauration de momies animales
}

An example of conservation - restoration of animal mummies

\section{Aurélie Paillier}

\section{OpenEdition}

\section{Journals}

Édition électronique

URL : http://journals.openedition.org/ocim/895

DOI : 10.4000/ocim.895

ISSN : 2108-646X

Éditeur

OCIM

Édition imprimée

Date de publication : 1 juillet 2011

Pagination : 21-26

ISSN : 0994-1908

Référence électronique

Aurélie Paillier, «Un exemple de conservation-restauration de momies animales », La Lettre de l'OCIM

[En ligne], 136 | 2011, mis en ligne le 01 juillet 2013, consulté le 19 avril 2019. URL : http://

journals.openedition.org/ocim/895; DOI : 10.4000/ocim.895

Tous droits réservés 
- la non-intervention : vouant ainsi l'objet à une dégradation progressive inéluctable à moyen voire à court terme ; - l'ntervention totale : imprégnation à cœur irréversible de l'objet :

- l'intervention limitée mais ciblée : compromis entre les deux précédentes. Intervenir le moins possible mais de manière à repousser l'échéance dans l'attente d'une solution ou d'une réponse peut-être plus adaptée à ce type d'objet.

L'étude menée a consisté à déterminer dans quelles mesures il serait possible de limiter les altérations par l'application d'un consolidant de surface d'une part et, d'autre part, de remettre en connexion les parties désolidarisées par l'application d'un adhésif, redonnant ainsi une lisibilité à « l'objet ».

\section{Le matériel et les méthodes}

Les deux poissons ayant fait l'objet de cette étude sont identifiés comme Lates niloticus (Perches du Nil). Ils sont datés de plus ou moins 2000 ans.

Le plus gros des deux spécimens a subi anciennement une infestation (Dermestidae non identifiés) qui a fragilisé sa structure/cohésion. Il présente de nombreuses lacunes (nageoires pelviennes, écailles) et certaines parties sont désolidarisées et fragmentées (nageoires caudale et dorsale), d'autres encore sont soulevées (plaques d'écailles) et/ou déplacées (plusieurs vertèbres).

Le second spécimen est moins lacunaire et anatomiquement presque complet. Cependant, il présente un état de surface extrêmement fragile : des fissures, des écailles abrasées, des soulèvements, une plaque d'écaille détachée, des écailles manquantes au niveau du pédoncule caudal.

Il était important de connaître la composition physico-chimique de la peau et des écailles, les procédés de momification employés et la nature des substances utilisées. En effet, leur détermination était primordiale, car l'adhésif et le consolidant ne doivent pas interférer avec la composition physico-chimique du substrat, d'où l'intérêt de mener en amont une étude à la fois théorique et pratique. Une fois celle-ci achevée, différents adhésifs (résine + solvant) et consolidants (résine + solvant) ont été préparés et testés en fonction de paramètres préalablement définis (neutralité, élasticité, matité, pénétration limitée...).

Concernant les procédés égyptiens de momification des poissons, la littérature ${ }^{(3)}$ mentionne l'utilisation de Natron $\left(\mathrm{NaCl}, \mathrm{CaCO}_{3}, \mathrm{CaSO}_{4}\right)$ riche en substances salines. Des analyses et des tests (nitrate d'argent)(4) réalisés sur des échantillons de surface des poissons étudiés attestent la présence de l'élément Chlorure. Pour cette raison, il était primordial que le diluant des résines à tester n'interfère pas avec ce dernier, constituant des produits de momification.

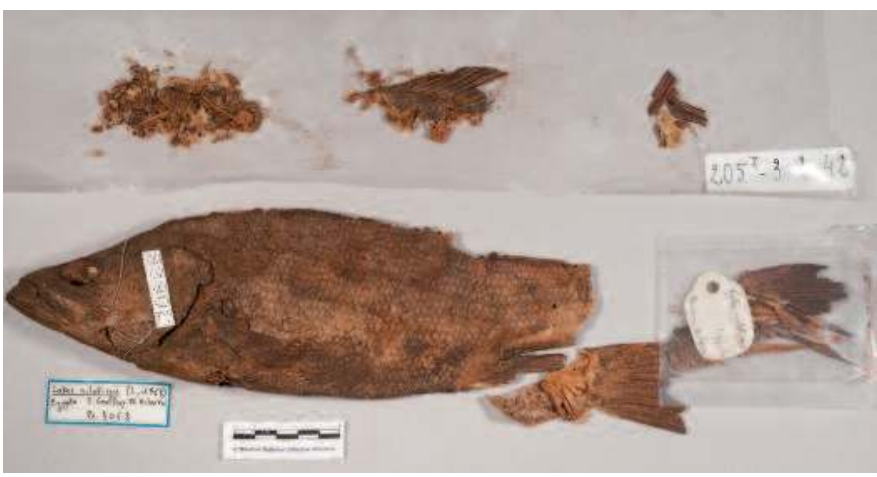

État avant restauration du spécimen Lates niloticus $N^{\circ}$ B-3068 côté gauche (๑) MNHN/C. Ferrara

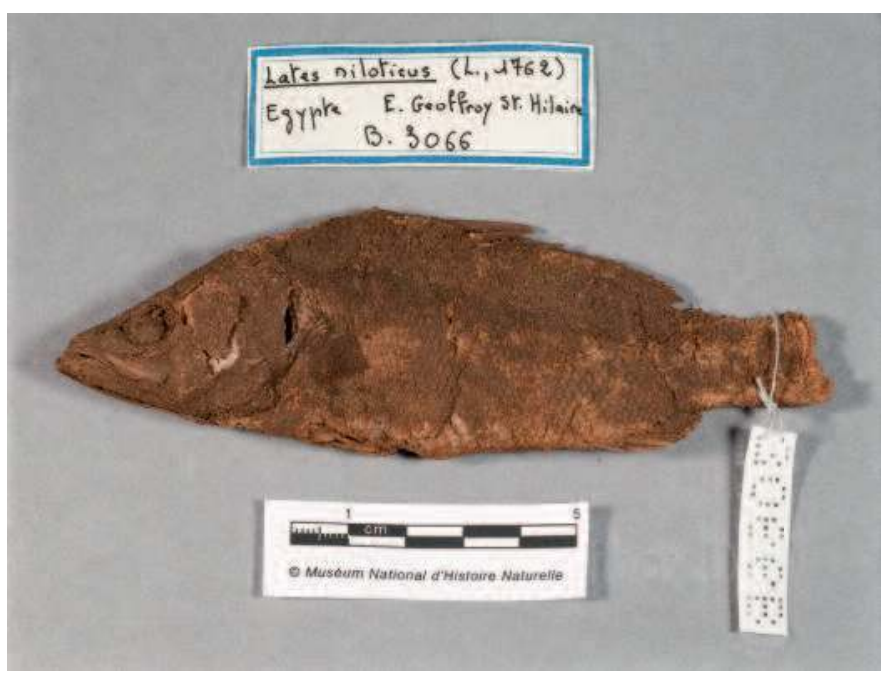

État avant restauration du spécimen Lates niloticus N B-3066 côté gauche (๑) MNHN/C. Ferrara

Parmi plusieurs solvants sélectionnés selon certains critères - volatilité élevée, tension superficielle (Ts) faible - l'acétone a donné de bons résultats au test sur du $\mathrm{NaCl}$ et a donc été choisie comme diluant des résines.

Plusieurs résines thermoplastiques - éthers de cellulose, vinyliques, acryliques - ont été sélectionnées en fonction de propriétés en accord avec les critères définis pour la mise en œuvre du consolidant et de l'adhésif. Afin d'étudier la viscosité, l'élasticité-résistance, la transparence, l'aspect (brillant ou mat), le temps d'évaporation, le temps de prise..., des solutions ont été préparées à des concentrations différentes pour chaque résine.

Une petite quantité de chaque adhésif obtenu a été appliquée en couche fine sur des lames porte-objets à concentrations diverses pour procéder aux diverses observations. Seuls les adhésifs répondant le mieux aux critères souhaités pour la consolidation de surface et le collage des parties désolidarisées, ont été sélectionnés.

Suite à cette sélection, il fallait tester le comportement des adhésifs retenus sur un matériau proche de la nature 
du substrat des momies (poisson séché). Les méthodes de mise en œuvre ont été également testées. Le traitement de conservation-restauration des deux momies de poissons a alors pu être réalisé.

L'Elvacite 2046 (résine acrylique) concentré à $20 \%$ dans l'acétone a été choisi comme consolidant et à $30 \%$ dans l'acétone comme adhésif. La consolidation des soulèvements et de certaines fissures s'est fait par doublage au papier Japon Tengujo $\left(6 \mathrm{~g} / \mathrm{m}^{2}\right)$ couplé à l'adhésif à $30 \%$ dans l'acétone (appliqué à la seringue) mais également par infiltration, à la seringue, sous les écailles. Le collage des parties désolidarisées s'est fait par infiltration d'Elvacite 2046 à $20 \%$ à la jonction des parties remises en connexion (nageoires dorsale et caudale). Les quatre vertèbres désolidarisées ont pu, quant à elles, être collées ensemble par couplage de papier Japon imbibé d'acétone puis adjonction, à la seringue, de

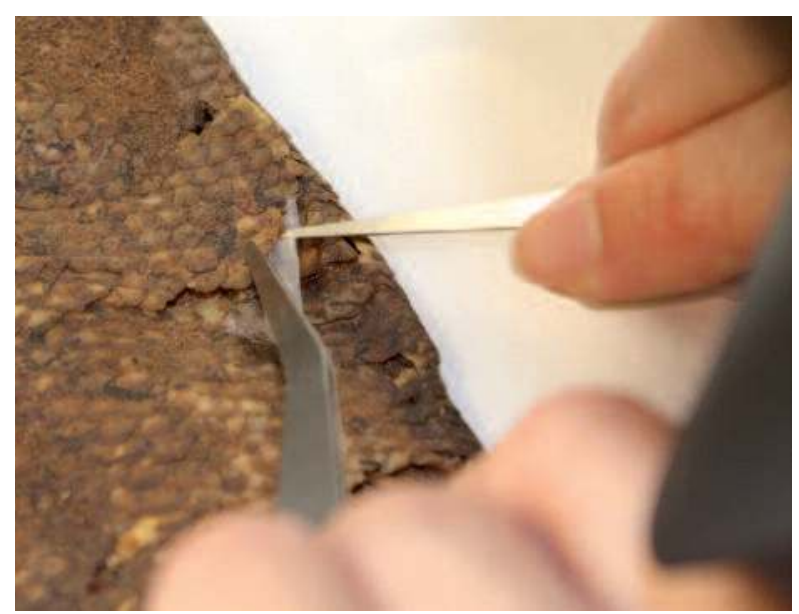

Insertion de papier Japon sous soulèvement du spécimen Lates niloticus $\mathrm{N}^{\circ} \mathrm{B}-3068$ (c) MNHN/J. Corny

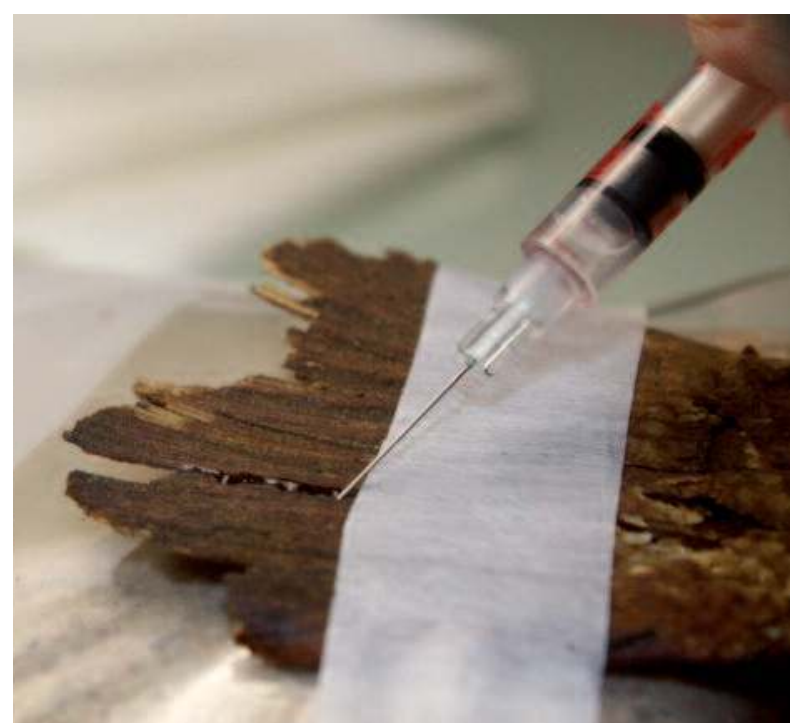

Infiltration à la seringue, à la jonction des deux parties de la nageoire caudale du spécimen Lates niloticus $\mathrm{N}^{\circ}$ B-3068 (c) MNHN/M. Cataldi
Paraloïd B72 à $20 \%$, qui a fait ses preuves dans le collage des os. Cette opération est intéressante à plusieurs titres :

- elle renforce la structure interne de la partie postérieure du corps ;

- elle met en évidence le rôle de support des plaques d'écailles qui ont tendance à s'affaisser en raison de la disparition de la structure interne causée par les insectes.

La même opération a été réalisée pour la remise en connexion et le collage des quatre vertèbres à la colonne vertébrale et à la plaque hypurale de la nageoire caudale. L'insertion de papier Japon au niveau du pédoncule caudal permet d'assurer plusieurs fonctions :

- une fonction tampon entre la colonne vertébrale et les plaques d'écailles qui vont être repositionnées ;

- une fonction support de collage, qui permet aussi de consolider la structure interne.

Aux quelques endroits où le papier Japon est visible, une retouche a été réalisée à l'aquarelle de manière à ne pas perturber la lisibilité globale.

\section{Les résultats}

Les deux momies de poissons ont retrouvé une certaine lisibilité et une certaine cohésion. Cependant, elles demeurent très fragiles.

Après les interventions de conservation-restauration sur le $\mathrm{N}^{\circ} \mathrm{B}-3068$, le résultat obtenu est satisfaisant dans la mesure où il a retrouvé une bonne lisibilité anatomique et une solidité osseuse qui lui redonnent une certaine cohésion. En effet, la consolidation effectuée sur cette momie est à la fois structurelle et de surface.

L’intervention pratiquée limite les dégradations et altérations mécaniques, mais n'agit pas sur les processus de dégradation physico-chimique liés aux variations de température et d'hygrométrie. Pour les limiter, il est primordial que ces matériaux organiques secs soient stockés dans un lieu stable au niveau de la température et de l'hygrométrie afin d'éviter le développement de micro-organismes favorisant la reprise du processus de décomposition.

Le résultat obtenu après les interventions de conservation restauration sur le $\mathrm{N}^{\circ} \mathrm{B}$-3066 n'est pas complètement satisfaisant, dans la mesure où il n'a été possible de limiter le délitement de surface des écailles que dans les zones les plus abîmées. En effet, pour une limitation complète du délitement de surface, il aurait fallu réaliser une imprégnation à cœur irréversible, non envisageable dans l'état actuel, car celle-ci modifierait à jamais la texture et l'aspect même de l'objet et le rendrait postérieurement non analysable. Les opérations effectuées étaient nécessaires, mais sont loin d'être suffisantes et ne font que repousser l'échéance de la réduction en poussière de l'objet. 


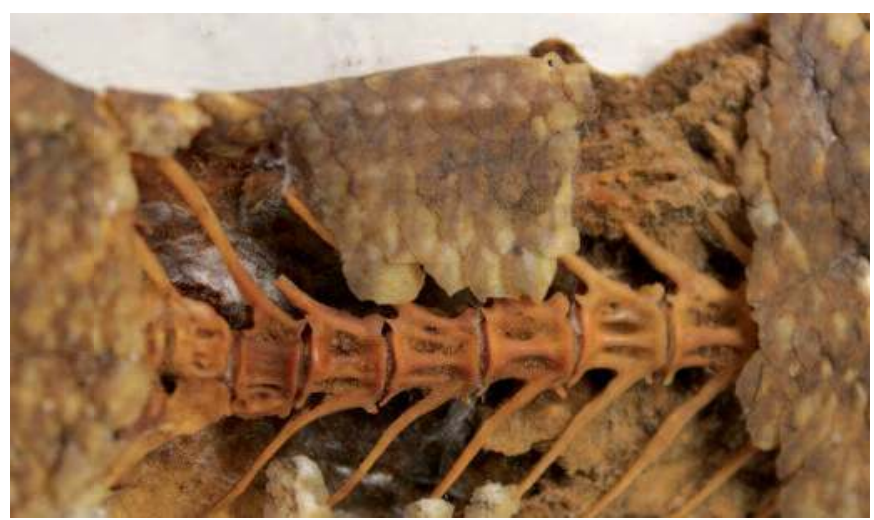

Vertèbres du spécimen Lates niloticus $N^{\circ}$ B-3068 après collage à la colonne vertébrale et à la plaque hypurale ○ MNHN/A. Paillier

Les deux momies ont fait l'objet d'une intervention limitée mais ciblée. Même si la réversibilité est une constante recherchée en conservation-restauration dans l'absolu, elle n'est pas toujours possible réellement. C'est un subtil mélange entre la théorie et la pratique.

La non-réversibilité de certaines interventions a été dans notre cas une décision réfléchie et nécessaire à la conservation des deux spécimens.

En effet, « la matière se dégradant selon des modalités et des rythmes qui dépendent de sa nature, il faut apprendre à identifier ces rythmes, puis adopter les démarches répondant précisément à ces risques »(Cuisin, 2005, p. 4). Il est important de se " poser les questions du sens de ces objets » et «des limites dans lesquelles doit s'exercer cette ouvre de préservation » (Cuisin, 2005, p. 4).

"S'il n'y a plus de matière, il n'y a plus de sens. [...] Il semble qu'il vaille mieux préserver ce qui peut l'être que de devoir tout perdre au nom d'une rigueur qui n'a pas de fondement raisonné. Préserver ce qui peut l'être encore, c'est vouloir faire le choix d'une valorisation scientifique et patrimoniale prolongée » (Cuisin, 2005, p. 8).

"Préserver et dans ce cadre restaurer, c'est contribuer à retarder l'usure de cette matière, pas à n'importe quel prix, ni en faisant n'importe quoi; mais en agissant avec la finalité de l'intervention constamment présente à l'esprit » (Cuisin, 2005, p. 7). «Sawver le spécimen d'une dégradation intense inéluctable et d'une disparition prématurée " (Cuisin, 2005, p. 12) est primordial.

Les procédés de momification égyptiens, les conditions d'enfouissement et du climat ont permis aux momies de poissons de parvenir jusqu'à nous dans un état de conservation exceptionnel si l'on considère leur âge (plus ou moins 2000 ans). Néanmoins, à compter de leur excavation jusqu'à aujourd'hui, des facteurs de nature diverse ont entrainé des altérations sur celles-ci et les ont grandement fragilisées. Les momies, bien que différentes des naturalia de par leurs composants et leur « conception », sont avant tout constituées de matière organique, par conséquent, elles sont amenées à disparaître.

"Comment préserver les valeurs de ces objets dans les conditions les plus intègres et le plus longtemps possible? Quelles interventions effectuer pour satisfaire à ces objectifs ? " (Cuisin, 2005, p. 5).

Dans notre cas, les actions de conservation préventive ne suffisaient pas à garantir leur longévité, d'où la nécessité de procéder à des interventions de restauration même si certaines ne sont pas totalement réversibles.

"Conserver, c'est choisir de faire durer un objet pour le transmettre à travers le temps et les générations, la restauration intervenant pour pallier les déficiences des mesures conservatoires. Cet effort [...] impose, pour avoir du sens, de porter sur l'authentique»(Cuisin, 2005, p. 7).

Une étude physico-chimique plus poussée en collaboration avec des chimistes ayant un domaine de compétence correspondant, pourrait être intéressante afin de voir s'il serait possible d'interrompre les processus entraînant des dégradations de cet ordre.

La conception, dans des matériaux neutres, de boîtes de stockage est indispensable et prolongera leur conservation à long terme. D'une part en limitant les manipulations des
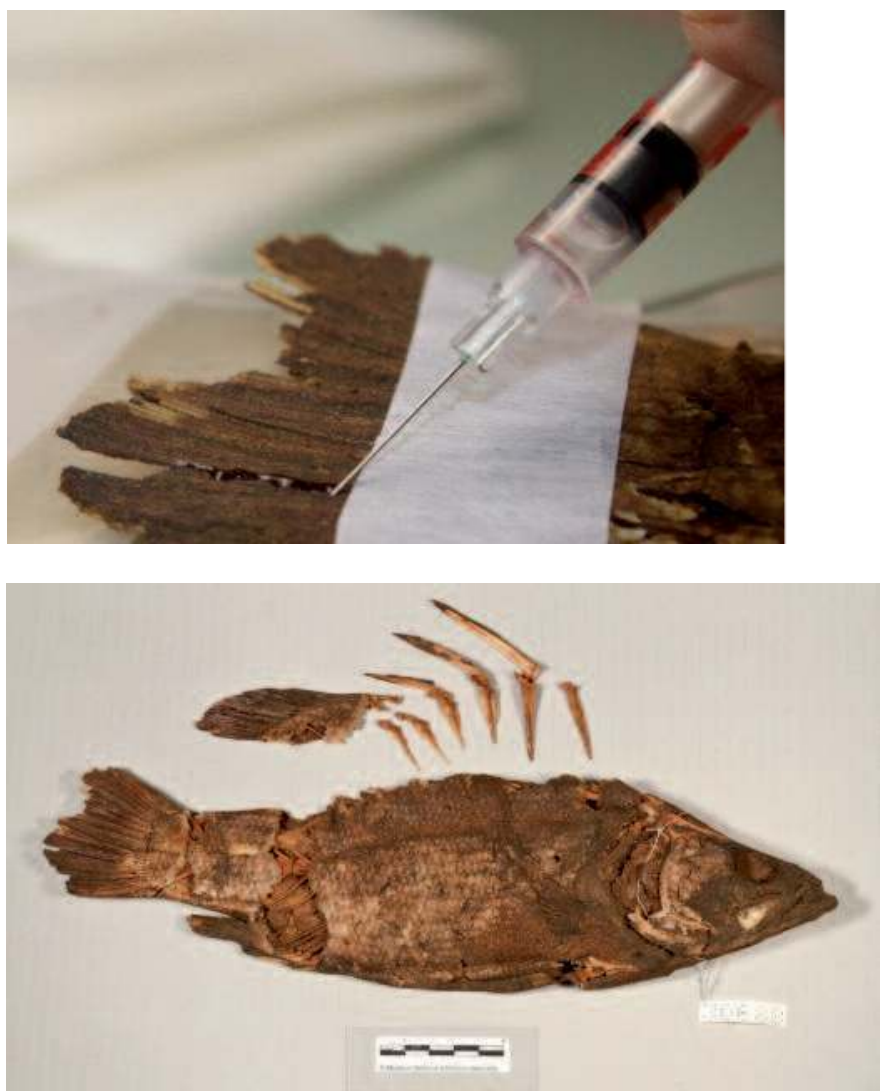

Les côtés gauche et droit du spécimen Lates niloticus $\mathrm{N}^{\circ} \mathrm{B}-3068$ après restauration ( $\mathrm{MNHN} / \mathrm{C}$. Ferrara 
spécimens, en offrant la possibilité de les sortir aisément sur un plateau, pour leur exposition par exemple, ou encore lors d'autres investigations ou opérations de conservation restauration. D'autre part en les protégeant des chocs éventuels lors de manipulations et de transports. Ces boîtes s'ouvrent sur un des grands côtés afin de pouvoir sortir la momie sans avoir besoin de la manipuler ni de la soulever.

\section{Conclusion}

L'identification et la mise en œuvre d'un consolidant de surface et d'un adhésif appliqués sur deux poissons momifiés égyptiens a été rendue possible grâce à toutes les études menées en amont : historique, physico-chimique, analyses, constat d'état, tests de compatibilité...

Dans le cas de notre étude, l'utilisation d'Elvacite 2046 en solution dans l'acétone a donné des résultats assez satisfaisants. Cependant, en l'absence de recul, et de données sur l'évolution possible à long terme de ces traitements sur ce type « d'objet », il convient de rester prudent. Si des opérations similaires de conservation-restauration devaient être menées sur des poissons momifiés de même espèce ou non, la compatibilité de l'Elvacite 2046 devrait être vérifiée. L'application de l'adhésif/consolidant identifié et mis en œuvre sur deux poissons momifiés égyptiens pourrait être envisagée sur d'autres types d'animaux momifiés à condition de procéder en amont à des tests de faisabilité.

Partis sceptiques sur ce qui pouvait être fait en conservation-restauration sur les deux spécimens momifiés, les Ichtyologues sont arrivés complètement convaincus des interventions qui ont été réalisées. En effet, les deux poissons ont retrouvé une lisibilité indéniable, en particulier pour le plus gros d'entre eux. Stockés dans de bonnes conditions, ils peuvent être présentés sans manipulation directe, et transportés sans risques.

\section{Notes}

(1) Ramenés d'Égypte par Étienne Geoffroy Saint-Hilaire lors de la campagne de Napoléon Bonaparte (1798-1801). Concernant l'historique se référer à : Paillier, A. Identification et mise en cuuvre d'un consolidant de surface et d'un adhésif appliqués sur deux poissons momifiés égyptiens. Travail de diplôme, HECR-Arc, La Chaux-de-Fonds (CH), 2010, pp. 1239, non publié et à la bibliographie non exhaustive.

(2) L'étude présentée dans cet article a été réalisée dans le cadre d'un travail de diplôme de conservation-restauration d'objets archéologiques et ethnographiques de la HECR-Arc, La Chaux-de-Fonds $(\mathrm{CH})$ :

Paillier, A. Identification et mise en œuvre d'un consolidant de surface et d'un adhésif appliqués sur deux poissons momifiés égyptiens. Travail de diplôme, HECR-Arc, La Chaux-de-Fonds (CH), 2010, 250 p., non publié. Ce travail de diplôme a été réalisé au MNHN de Paris, dans le laboratoire de conservation des momies du musée de l'Homme.

(3) Dunand, F. et Lichtenberg, R. Momies d'Égypte et d'ailleurs, La mort refusée. Éditions du Rocher, 2002, pp. 17-19.

Gaillard, C. et Daressy, G. Catalogue général des Antiquités Égyptiennes du musée du Caire, Nos 29501-29733 et 29751-29834. La Faune momifiée de l'antique Égypte. Imprimerie de l'institut français d'archéologie orientale, Le Caire, 1905, pp. 72-73.

Ikram, S. Divine creatures : animal mummies in ancient Egypt. American University in Cairo Press, 2005, pp. xvii, xviii, 41.

(4) Les analyses effectuées sur les poissons ont été réalisées sur le site du MNHN, dans différents services : laboratoire de Spectroscopie Infra Rouge, laboratoire de Chimie et Biochimie des substances naturelles...

\section{Bibliographie}

Blainville, H.-M. D. de. Cuvier et Geoffroy Saint-Hilaire : Biographies scientifiques. Paris : Librairie J.B. Baillière et fils, 1890, 446 p.

Brier, B. et Bennett, M.L.V. Autopsies on Fish Mummies : Possible Identification of the Classical Phagrus, The Journal of Egyptian Archaeology, vol. 65, 1979, pp. 128-133.

Cahn, T. La vie et l'œuvre d'Étienne Geoffroy Saint-Hilaire. Paris : Presses Universitaires de France, 1962, 318 p.

Cuisin, J. Être ou ne plus être : quelle restauration pour les naturalia? Conservation-Restauration des Biens Culturels (CRBC), n²3, 2005, pp. 3-15.

Dunand, F. et Lichtenberg, R. Momies d'Égypte et d'ailleurs, La mort refusée. Éditions du Rocher, 2002, 270 p.

Gaillard, C. et Daressy, G. Catalogue général des Antiquités Égyptiennes du musée du Caire, Nos 29501-29733 et 29751-29834. La Faune momifiée de l'antique Égypte. Le Caire : Imprimerie de l'institut français d'archéologie orientale, 1905, $159 \mathrm{p}$.

Geoffroy Saint-Hilaire, E. L'expédition d'Égypte : 1798-1802. ClermontFerrand : Éditions Paléo, 2000. Classiques de l'histoire des sciences, 184 p.

Geoffroy Saint-Hilaire, E. Notices sur les travaux du citoyen Geoffroy Saint-Hilaire, professeur de Zoologie au Muséum d'Histoire Naturelle. [s.n.], an 11 (1803).

Geoffroy Saint-Hilaire, I. Vie, travaux et doctrine scientifique d'Étienne Geoffroy Saint-Hilaire; par son fils M. Isidore Geoffroy Saint-Hilaire. Paris : P. Bertrand ; Strasbourg : Veuve Levrault, 1847, 479 p.

Ikram, S. Divine creatures : animal mummies in ancient Egypt. American University in Cairo Press, 2005, pp. xvii, xviii, 41. 
Kury, L. Histoire naturelle et voyages scientifiques : (1780-1830). Paris, Montréal, Budapest : L’Harmattan, 2001, 236 p.

Mannes, G. Le Grand Ouvrage: Description de l'Égypte, ou recueil des observations et des recherches qui ont été faites en Égypte pendant l'expédition de l'armée française, publié par les ordres de sa majesté l'Empereur Napoléon le Grand: La Bibliothèque Grand-Ducale en visite à la Bibliothèque Nationale. Exposition à la Bibliothèque nationale du Luxembourg du 12 décembre 2003 au 31 mars 2004. Luxembourg : Bibliothèque nationale du Luxembourg, 2003, 268 p.
Paillier, A. Identification et mise en œuvre d'un consolidant de surface et d'un adhésif appliqués sur deux poissons momifiés égyptiens, travail de diplôme, HECR-Arc, La Chaux-de-Fonds (CH), 2010, 250 p., non publié.

Rolland-Villemot, B. Les spécificités de la conservation-restauration des collections ethnographiques, la Lettre de l’OCIM, n56, 1998, pp. 15-19. 